\title{
Inequalities Characterizing Linear-Multiplicative Functionals
}

\author{
Włodzimierz Fechner \\ Institute of Mathematics, University of Silesia, Bankowa 14, 40-007 Katowice, Poland \\ Correspondence should be addressed to Włodzimierz Fechner; fechner@math.us.edu.pl \\ Received 14 October 2014; Accepted 13 January 2015 \\ Academic Editor: Bruce A. Watson \\ Copyright (C) 2015 Włodzimierz Fechner. This is an open access article distributed under the Creative Commons Attribution \\ License, which permits unrestricted use, distribution, and reproduction in any medium, provided the original work is properly \\ cited. \\ We prove, in an elementary way, that if a nonconstant real-valued mapping defined on a real algebra with a unit satisfies certain \\ inequalities, then it is a linear and multiplicative functional. Moreover, we determine all Jensen concave and supermultiplicative \\ operators $T: C(X) \rightarrow C(Y)$, where $X$ and $Y$ are compact Hausdorff spaces.
}

Let $\mathscr{A}$ be an algebra with unit $\mathbf{1} \in \mathscr{A}$ over the field of real numbers. A map $\phi: \mathscr{A} \rightarrow \mathbb{R}$ is midpoint convex or Jensen convex, if the inequality

$$
\phi\left(\frac{f+g}{2}\right) \leq \frac{\phi(f)+\phi(g)}{2}
$$

holds for every $f, g \in \mathscr{A}$. If this inequality holds in reverse, then a map $\phi: \mathscr{A} \rightarrow \mathbb{R}$ is midpoint concave or Jensen concave. Further, a map $\phi: \mathscr{A} \rightarrow \mathbb{R}$ is supermultiplicative, if the inequality

$$
\phi(f \cdot g) \geq \phi(f) \cdot \phi(g)
$$

holds for every $f, g \in \mathscr{A}$.

First, we show that a nonconstant mapping $\phi: \mathscr{A} \rightarrow \mathbb{R}$ is midpoint concave and supermultiplicative if and only if it is a linear and multiplicative functional. In the proof we follow some ideas of J. X. Chen and Z. L. Chen [1], Dhombres [2], Ercan [3], Gusic [4], Radulescu [5], and Volkmann $[6,7]$.

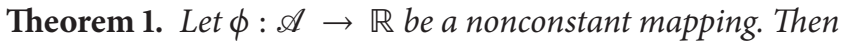
$\phi$ is midpoint concave and supermultiplicative if and only if it is a linear and multiplicative functional.

Proof. The "if" condition is straightforward; thus we will prove the "only if" condition. We will divide the proof into four steps.
Step $1(\phi(0)=0)$. Suppose for a contradiction that $\phi(0) \neq 0$. Note that for every $f \in \mathscr{A}$ we have

$$
\phi(0) \geq \phi(0) \cdot \phi(f) .
$$

Thus, in particular, $\phi(0) \geq \phi(0)^{2}>0$ and, consequently, we deduce that $\phi(f) \leq 1$ for all $f \in \mathscr{A}$. Next, for each $f \in \mathscr{A}$ one can note the following estimate:

$$
\phi(f)^{2} \leq \phi\left(f^{2}\right) \leq 1 .
$$

Thus, we arrive at

$$
-1 \leq \phi(f) \leq 1, \quad f \in \mathscr{A} .
$$

Let us define

$$
\begin{aligned}
& a=\inf \{\phi(f): f \in \mathscr{A}\}, \\
& b=\sup \{\phi(f): f \in \mathscr{A}\} .
\end{aligned}
$$

Therefore, we can write

$$
-1 \leq a \leq \phi(f) \leq b \leq 1, \quad f \in \mathscr{A} .
$$

Moreover, since $\phi(0)>0$, then $b>0$.

Since $\phi$ is nonconstant, then $a<b$. Therefore, we can pick some $f, g \in \mathscr{A}$ such that

$$
\phi(g)-\phi(f)>\frac{b-a}{2}
$$


Utilizing the fact that $\phi$ is midpoint concave, we obtain the inequality

$$
\phi(f)=\phi\left(\frac{2 f-g+g}{2}\right) \geq \frac{\phi(2 f-g)+\phi(g)}{2} .
$$

From the previous estimates we get

$$
\phi(g)-\phi(2 f-g) \geq 2[\phi(g)-\phi(f)]>2 \frac{b-a}{2}=b-a,
$$

a contradiction.

Step 2 ( $\phi$ is an odd mapping). Let $f \in \mathscr{A}$. Then,

$$
\begin{gathered}
\phi\left(f^{2}\right)=\phi(f \cdot f) \geq \phi(f)^{2}, \\
\phi\left(f^{2}\right)=\phi((-f) \cdot(-f)) \geq \phi(-f)^{2}, \\
\phi\left(-f^{2}\right)=\phi(f \cdot(-f)) \geq \phi(f) \phi(-f), \\
\phi\left(f^{2}\right)+\phi\left(-f^{2}\right) \leq 2 \phi\left(\frac{f^{2}-f^{2}}{2}\right)=2 \phi(0)=0 .
\end{gathered}
$$

Putting these together we get

$$
\begin{aligned}
0 & \leq[\phi(f)+\phi(-f)]^{2}=\phi(f)^{2}+2 \phi(f) \cdot \phi(-f)+\phi(-f)^{2} \\
& \leq 2 \phi\left(f^{2}\right)+2 \phi\left(-f^{2}\right) \leq 0 .
\end{aligned}
$$

This means that $\phi$ is odd.

Step 3 ( $\phi$ is additive and multiplicative). Utilizing Step 2 we obtain

$$
\begin{aligned}
\phi\left(\frac{f+g}{2}\right) & =-\phi\left(\frac{-f-g}{2}\right) \leq-\frac{\phi(-f)+\phi(-g)}{2} \\
& =\frac{\phi(f)+\phi(g)}{2}, \\
\phi(f \cdot g) & =-\phi(-f \cdot g) \leq-\phi(-f) \cdot \phi(g) \\
& =\phi(f) \cdot \phi(g),
\end{aligned}
$$

for all $f, g \in \mathscr{A}$. Thus $\phi$ is midpoint convex and multiplicative. Therefore, by Step 2 we infer that $\phi$ is additive.

Step 4 ( $\phi$ is $\mathbb{R}$-homogeneous). Observe that $\phi(\mathbf{1})=\phi(\mathbf{1} \cdot \mathbf{1})=$ $\phi(\mathbf{1})^{2}$. Therefore, either $\phi(\mathbf{1})=0$ or $\phi(\mathbf{1})=1$. In the first case we get that $\phi(f)=\phi(f \cdot \mathbf{1})=0$ for every $f \in \mathscr{A}$. Since $\phi$ is nonconstant, we get a contradiction. Therefore $\phi(\mathbf{1})=1$.

Define a map $\psi: \mathbb{R} \rightarrow \mathbb{R}$ by $\psi(\lambda)=\phi(\lambda \cdot \mathbf{1})$. Clearly, $\psi$ is additive and multiplicative and $\psi(1)=1$. Therefore $\psi(\lambda)=\lambda$ for all $\lambda \in \mathbb{R}$. Now, fix arbitrary $\lambda \in \mathbb{R}$ and $f \in \mathscr{A}$. We have

$$
\phi(\lambda f)=\phi((\lambda \cdot \mathbf{1}) \cdot f)=\psi(\lambda) \phi(f)=\lambda \phi(f) .
$$

Remark 2. Theorem 1 can be easily generalized to even more general spaces. An inspection of the proof shows that if we assume that $\mathscr{A}$ is an arbitrary ring (not necessarily unital), then every nonconstant midpoint concave and superadditive mapping $\phi: \mathscr{A} \rightarrow \mathbb{R}$ is additive and multiplicative.

It is clear that if $\phi: \mathscr{A} \rightarrow \mathbb{R}$ is a constant supermultiplicative mapping equal to some $c \in \mathbb{R}$, then $0 \leq c \leq 1$. Using this observation we derive an immediate corollary describing Jensen concave and supermultiplicative functions on the real line.

Corollary 3. All functions $f: \mathbb{R} \rightarrow \mathbb{R}$ which fulfill the system

$$
\begin{gathered}
f\left(\frac{x+y}{2}\right) \geq \frac{f(x)+f(y)}{2}, \\
f(x y) \geq f(x) f(y),
\end{gathered}
$$

for all $x, y \in \mathbb{R}$ are of one of the following forms:

(i) $f(x)=x$ for all $x \in \mathbb{R}$,

(ii) $f(x)=c$ for all $x \in \mathbb{R}$,

with some $c \in[0,1]$.

We will provide two easy examples showing that if one reverse the inequality sign in one inequality of (15), then the assertion of Corollary 3 remains no longer valid, even if the remaining inequality holds with an equality.

Example 4. The absolute value of a real number satisfies the second inequality of (15) (with equality) and the reverse of the first one.

Every constant mapping equal to a negative number satisfies the converse to the second inequality of (15) and is both Jensen convex and Jensen concave. More generally, every real mapping with nonpositive values is submultiplicative. Therefore, to show that there are nonconstant solutions one can take any nonpositive convex mapping (e.g., $f(x)=$ $-\exp (x)$ for $x \in \mathbb{R})$.

In our next result we apply Theorem 1 to determine all Jensen concave and supermultiplicative operators $T$ : $C(X) \rightarrow C(Y)$, where $X$ and $Y$ are compact Hausdorff spaces. Symbols $C(X)$ and $C(Y)$ denote the spaces of all real continuous functions defined on $X$ or $Y$, respectively. These spaces are furnished with standard algebraic operations, pointwise partial order, and the supremum norm.

Theorem 5. Assume that $X$ and $Y$ are compact Hausdorff spaces and let $T: C(X) \rightarrow C(Y)$ be an arbitrary mapping. Then $T$ is a Jensen concave and supermultiplicative operator if and only if there exist a clopen (i.e., closed and open) set $B \subseteq Y$, function $\varphi: Y \rightarrow X$, and continuous function $c: Y \rightarrow[0,1]$ such that $\varphi$ is continuous on $B$ and

$$
T(f)(x)= \begin{cases}(f \circ \varphi)(x), & x \in B, \\ c(x), & x \in X \backslash B\end{cases}
$$

for every $f \in C(X)$. 
Proof. The "if" condition is straightforward to check. Therefore, we will prove the "only if" condition.

Denote

$$
\begin{gathered}
A=\{x \in Y: T(0)(x) \neq 0\}, \\
B_{0}=X \backslash A=\{x \in Y: T(0)(x)=0\} .
\end{gathered}
$$

For a fixed $x \in Y$ define a map $\phi: C(X) \rightarrow \mathbb{R}$ by the formula

$$
\phi(f)=T(f)(x), \quad f \in C(X) .
$$

It is clear that $\phi$ is a Jensen concave and supermultiplicative functional. Therefore, we can utilize Theorem 1. If $x \in A$, then $\phi$ is constant. If $\phi$ is constant, say $\phi(f)=c$ for all $f \in C(X)$, then we have $0<c \leq 1$. Note that the constant $c$ may depend upon $x \in A$. Therefore, there exists a function $c_{0}: A \rightarrow$ $(0,1]$ such that $\left.T(f)\right|_{A}=c_{0}$ with every $f \in C(X)$. Let us put $c=T(0)$. Note that $c$ and $c_{0}$ agree on $A$ and moreover $T\left(0^{2}\right) \geq T(0)^{2} \geq T(0)$, so $c \geq 0$ and $c(x)=0$ for $x \in B_{0}$.

Now, fix some $x \in B_{0}$ and consider the function $\phi$ : $C(X) \rightarrow \mathbb{R}$ defined by formula (18) above. By Theorem 1 we get that $\phi$ is a linear and multiplicative functional of the space $C(X)$. Therefore, either $\phi \equiv 0$ or there exists some (unique) point $x_{0} \in X$ (depending upon the choice of $x \in Y$ ) such that

$$
\phi(f)=f\left(x_{0}\right), \quad f \in C(X) .
$$

Let $B \subseteq B_{0}$ be the set on which the second case holds and denote $\varphi(x)=x_{0}$ for $x \in B$. Note that the case $\phi \equiv 0$ is covered in the assertion of our theorem, since $c(x)=0$ for $x \in B_{0}$. Therefore, formula (16) is fully proved.

We will show that $B$ is a clopen set. Let $f_{d} \in C(X)$ be any constant mapping equal to some $d$. Since $T\left(f_{d}\right)$ is a continuous mapping, according to formula (16), for every $d \in \mathbb{R}$ the following functions are continuous for every $d \in \mathbb{R}$ :

$$
T\left(f_{d}\right)(x)= \begin{cases}d, & x \in B, \\ c(x), & x \in X \backslash B .\end{cases}
$$

Observe that the characteristic map of the set $B$ can be written as $\chi_{B}=T\left(f_{2}\right)-T\left(f_{1}\right)$. Consequently, $\chi_{B}$ is continuous, because $T\left(f_{1}\right)$ and $T\left(f_{2}\right)$ are continuous. From this we get that $B$ is a clopen set.

To finish the proof we need to show that $\varphi$ is continuous on $B$. Note that $\varphi$ is not involved in the assertion of the theorem outside the set $B$. And the continuity of $\varphi$ on $B$ follows from the equality $T(f)=f \circ \varphi$ on $B$ and from the Urysohn's lemma, which implies that elements of $C(X)$ separate points of $X$.

If we assume additionally that the space $X$ is connected, then the form of operators in question can be specified further.

Corollary 6. Under assumptions of Theorem 5, if additionally the space $Y$ is connected, then $T$ is a Jensen concave and supermultiplicative operator if and only if either there exists a continuous function $\varphi: Y \rightarrow X$ such that

$$
T(f)=f \circ \varphi, \quad f \in C(X),
$$

or there exists a continuous function $c: Y \rightarrow[0,1]$ such that

$$
T(f)=c, \quad f \in C(X) .
$$

\section{Conflict of Interests}

The author declares that there is no conflict of interests regarding the publication of this paper.

\section{References}

[1] J. X. Chen and Z. L. Chen, "On supra-additive and supramultiplicative maps," Journal of Function Spaces and Applications, vol. 2013, Article ID 108535, 3 pages, 2013.

[2] J. Dhombres, "Sur les fonctions simultanément suradditives et surmultiplicatives," Comptes Rendus Mathématiques, vol. 5, no. 5, pp. 207-210, 1983.

[3] Z. Ercan, "A remark on supra-additive and supra-multiplicative operators on $C(X)$," Mathematica Bohemica, vol. 132, no. 1, pp. $55-58,2007$.

[4] I. Gusic, "A note on certain maps between ordered fields," Journal of Mathematical Inequalities, vol. 3, no. 4, pp. 657-661, 2009.

[5] M. Radulescu, "On a supra-additive and supra-multiplicative operator of $\mathrm{C}(\mathrm{X})$," Bulletin Mathématique de la Société des Sciences Mathématiques de Roumanie (Nouvelle Série), vol. 24, no. 72, pp. 303-305, 1980.

[6] P. Volkmann, "Sur un système d'équations fonctionnelles," Comptes Rendus Mathématiques (Mathematical Reports) des l'Académie des Sciences, vol. 4, pp. 155-158, 1982.

[7] P. Volkmann, "Sur les fonctions simultanément suradditives et surmultiplicatives," Bulletin Mathématique de la Société des Sciences Mathématiques de la République Socialiste de Roumanie. Nouvelle Série, vol. 28, no. 76, pp. 181-184, 1984. 


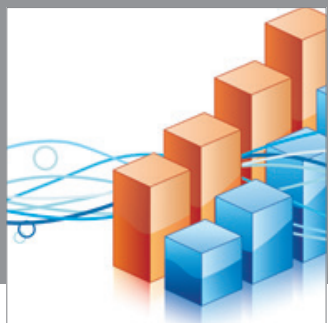

Advances in

Operations Research

mansans

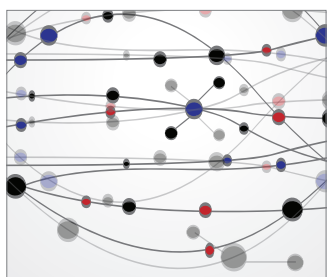

The Scientific World Journal
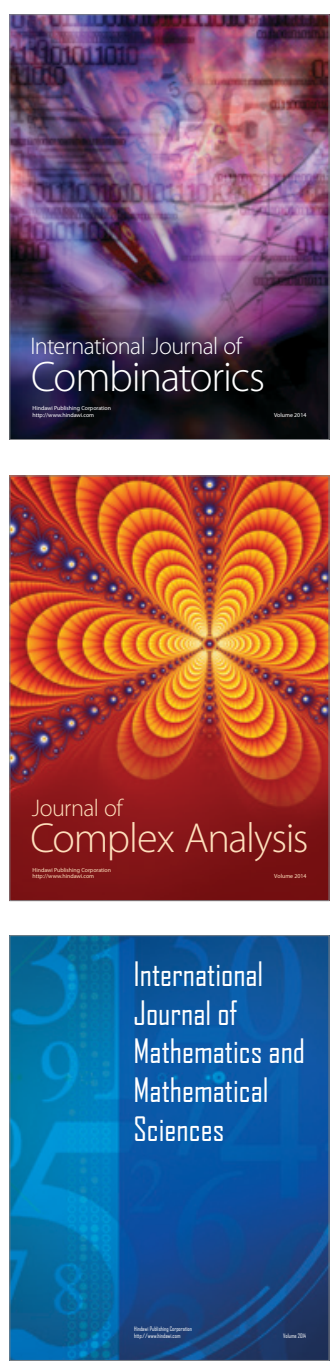
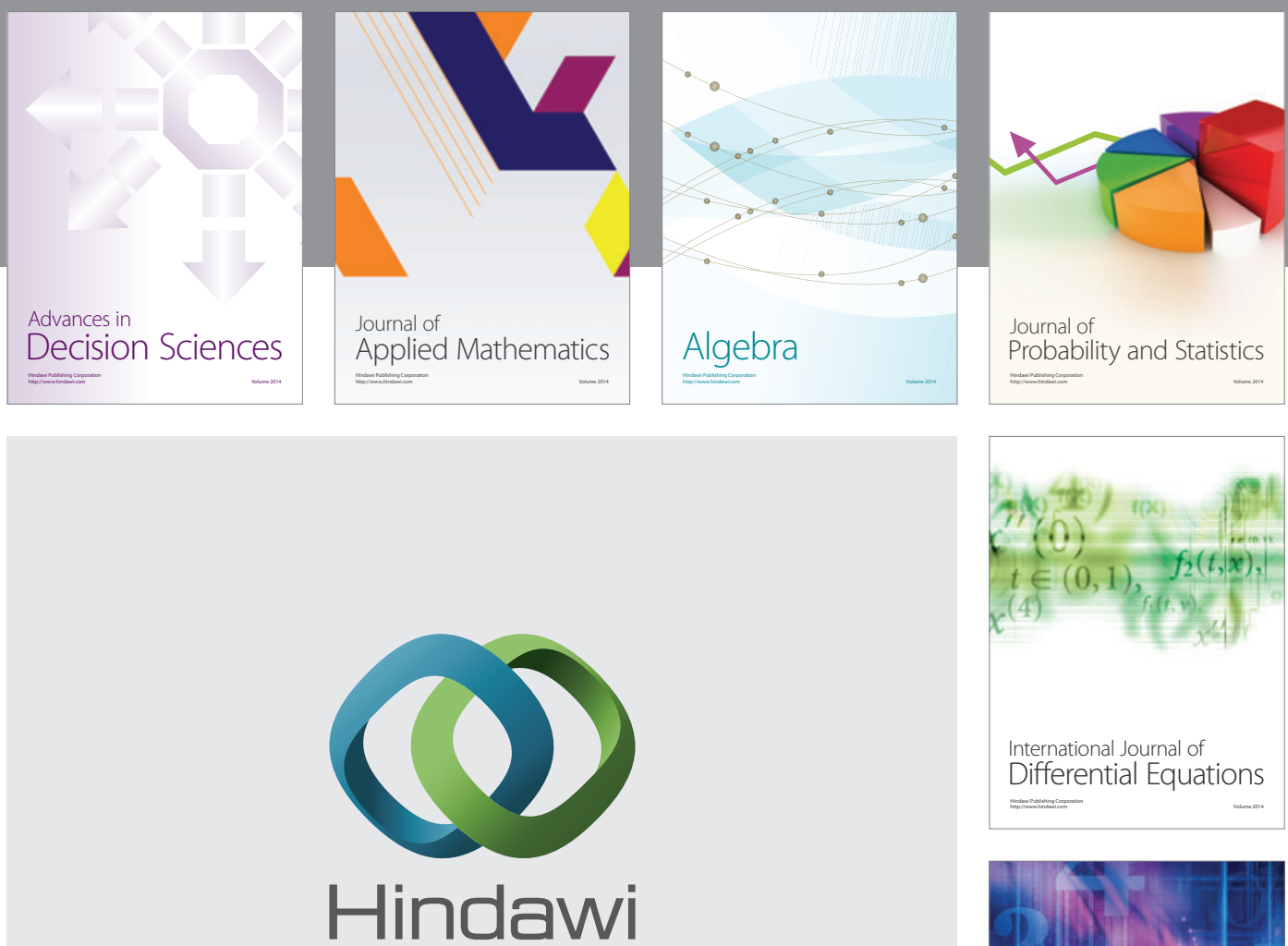

Submit your manuscripts at http://www.hindawi.com
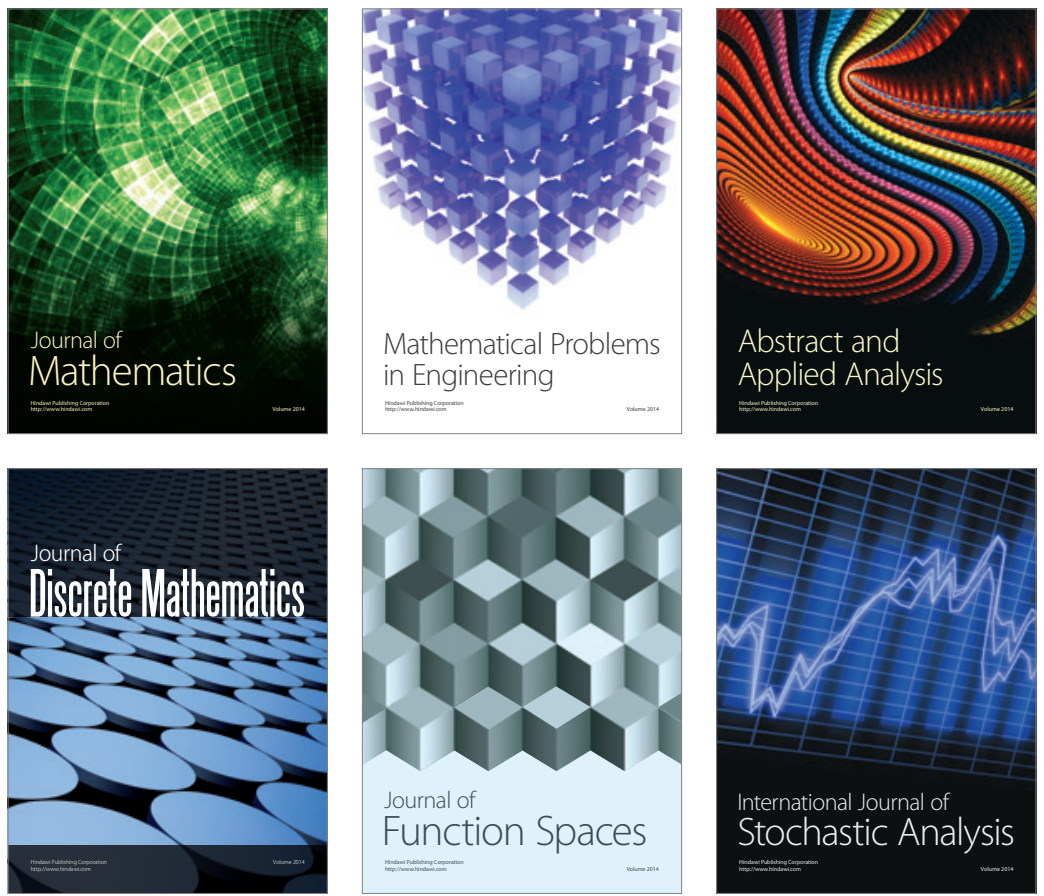

Journal of

Function Spaces

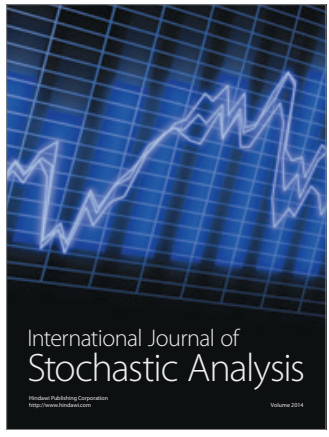

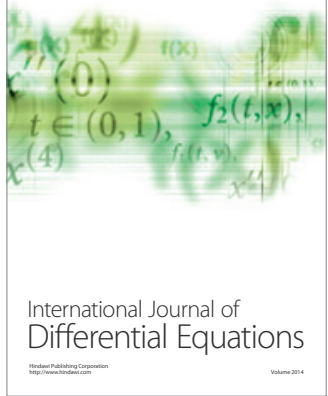
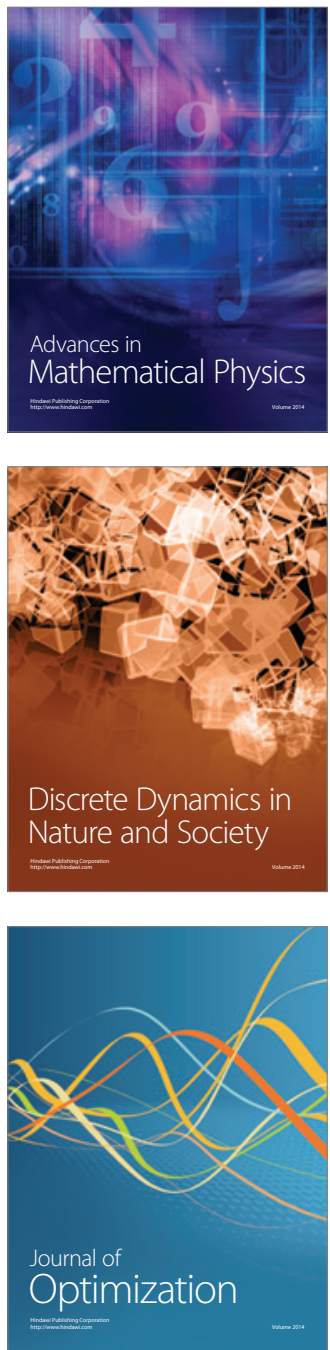